\title{
BMJ Open Trends in kidney function testing in UK primary care since the introduction of the quality and outcomes framework: a retrospective cohort study using CPRD
}

\author{
Benjamin Feakins, ${ }^{\oplus 1}$ Jason Oke, ${ }^{1}$ Emily McFadden, ${ }^{1}$ Jeffrey Aronson, ${ }^{1}$ \\ Daniel Lasserson, ${ }^{\circ, 3}$ Christopher O'Callaghan,, ${ }^{3,4}$ Clare Taylor, ${ }^{\oplus 1}$ Nathan Hill, ${ }^{5}$ \\ Richard Stevens, ${ }^{1}$ Rafael Perera ${ }^{1}$
}

To cite: Feakins B, Oke J, McFadden $\mathrm{E}$, et al. Trends in kidney function testing in UK primary care since the introduction of the quality and outcomes framework: a retrospective cohort study using CPRD. BMJ Open 2019;9:e028062. doi:10.1136/ bmjopen-2018-028062

- Prepublication history and additional material for this paper are available online. To view these files, please visit the journal online (http://dx.doi. org/10.1136/bmjopen-2018028062).

Received 22 November 2018 Revised 1 May 2019 Accepted 2 May 2019

Check for updates

(C) Author(s) (or their employer(s)) 2019. Re-use permitted under CC BY-NC. No commercial re-use. See rights and permissions. Published by BMJ.

For numbered affiliations see end of article.

Correspondence to

Dr Jason Oke;

jason.oke@phc.ox.ac.uk

\section{ABSTRACT}

Objectives To characterise serum creatinine and urinary protein testing in UK general practices from 2005 to 2013 and to examine how the frequency of testing varies across demographic factors, with the presence of chronic conditions and with the prescribing of drugs for which kidney function monitoring is recommended.

Design Retrospective open cohort study.

Setting Routinely collected data from 630 UK general practices contributing to the Clinical Practice Research Datalink.

Participants 4573275 patients aged over 18 years registered at up-to-standard practices between 1 April 2005 and 31 March 2013. At study entry, no patients were kidney transplant donors or recipients, pregnant or on dialysis.

Primary outcome measures The rate of serum creatinine and urinary protein testing per year and the percentage of patients with isolated and repeated testing per year.

Results The rate of serum creatinine testing increased linearly across all age groups. The rate of proteinuria testing increased sharply in the 2009-2010 financial year but only for patients aged 60 years or over. For patients with established chronic kidney disease (CKD), creatinine testing increased rapidly in 2006-2007 and 2007-2008, and proteinuria testing in 2009-2010, reflecting the introduction of Quality and Outcomes Framework indicators. In adjusted analyses, CKD Read codes were associated with up to a twofold increase in the rate of serum creatinine testing, while other chronic conditions and potentially nephrotoxic drugs were associated with up to a sixfold increase. Regional variation in serum creatinine testing reflected country boundaries.

Conclusions Over a nine-year period, there have been increases in the numbers of patients having kidney function tests annually and in the frequency of testing. Changes in the recommended management of CKD in primary care were the primary determinant, and increases persist even after controlling for demographic and patientlevel factors. Future studies should address whether increased testing has led to better outcomes.
Strengths and limitations of this study

- To date, this is the largest population study of trends in renal function testing in primary care.

- The data source did not permit us to ascertain why a kidney function test was performed.

- The transitioning of 'high risk' patients from primary to secondary care means the estimates in this study may be liable to underestimate the amount of testing performed in certain patient subgroups.

\section{INTRODUCTION}

Kidney function testing in primary care is used to diagnose and monitor chronic kidney disease (CKD). Testing is recommended at baseline and after initiation of some drugs such as antihypertensives. ${ }^{1}$ Kidney function is usually tested by measuring serum creatinine, and screening for glomerular disease is undertaken by measuring urine albumin or protein concentrations.

In 2002, the Kidney Disease Outcomes and Quality Initiative (K/DOQI) published clinical guidelines advocating that CKD be categorised into five stages. ${ }^{2}$ Two years later, these stages were adopted by the UK Quality and Outcomes Framework (QOF), which is a set of business rules for primary care that include financial incentives to regularly monitor and test certain subsets of patients and to record their data. ${ }^{3}$ The 2006-2007 financial year saw an extension to QOF that required general practitioners to maintain a register of patients with CKD stages $3-5 .{ }^{4}$ In 2008, the National Institute for Health and Care Excellence (NICE) recommended monitoring estimated glomerular filtration rate (eGFR) levels in high-risk patients. ${ }^{5}$ Then in the 2009-2010 financial year, a further QOF extension incentivised monitoring urinary markers of kidney disease (such as proteinuria) in patients on 
the CKD register. ${ }^{6}$ Current NICE recommendations on the frequency of testing are based on the underlying cause of CKD, previous test results, comorbidities and the treatments being used. Monitoring is recommended annually in patients with mild to moderate reductions in kidney function and every three months in patients with more advanced disease. ${ }^{1}$

National rates of kidney function testing and potential differences between different populations have not been characterised. In contrast, rates of kidney function testing in patients with diabetes have been well documented. A cohort study of adults with diabetes showed that under $13 \%$ had incomplete CKD screening, and just $4.4 \%$ had no serum creatinine measurement on record in the two and half years before the start of the study, whereas the albumin-creatinine ratio (ACR) was not monitored in $37 \%$ during the same period. ${ }^{7}$ Similarly, high frequencies of serum creatinine testing have been observed among patients with diabetes in studies looking at individual health regions, but with more variable levels of recording in patients without diabetes across different ages, genders and ethnic groups. ${ }^{8}$

There has been a dramatic increase in the use of laboratory testing over recent decades, particularly repeated testing or monitoring. ${ }^{9}{ }^{10}$ However, it is unclear whether this increase is appropriate and consistent with guideline recommendations or whether it represents overtesting. Appropriate testing of kidney function might be of value in planning management to slow the progression of the disease and, therefore, lead to tangible patient benefit. However, overuse of tests provides little patient benefit and adds to the financial burden of healthcare systems. A recent meta-analysis of the use of laboratory tests during the last 15 years showed that underuse of high-volume tests (such as creatinine) was more likely than overuse. ${ }^{11}$ A cross-sectional survey of US physicians' patterns of care in patients with CKD showed that $85 \%$ of physicians recommended one additional test, which was not recommended in the Kidney Disease Improving Global Outcomes (KDIGO) guidelines. ${ }^{12}$ These tests were most likely to be magnetic resonance angiography of renal arteries or serum protein electrophoresis, rather than blood or urinary measurements. ${ }^{13}$ It is, of course, possible that overuse and underuse may coexist, with some patients receiving more tests than indicated and other patients not receiving tests warranted by their clinical history, recent health and age.

Currently, the UK is the only nationalised and publicly funded health service that has introduced financial incentives to improve the quality of healthcare for patients with CKD. National guidelines in other countries also recommend quality standards for CKD care, including diagnosis, monitoring of renal function and control of cardiovascular risk factors. ${ }^{14}$ However, guideline bodies outside the UK have stopped short of implementing financial incentives for CKD care, and therefore, studying the impact of QOF in the UK can inform international efforts to improve outcomes for patients with CKD.
The aim of this study is to describe rates of kidney function testing since the introduction of the QOF in UK general practice. Specifically, we have examined the numbers of serum creatinine and proteinuria tests requested in each financial year during the nineyears from 2005 to 2013 by: age category, gender, ethnicity, index of multiple deprivation (IMD), Strategic Health Authority (SHA), CKD stage, the presence or absence of major comorbidities (such as diabetes, hypertension, cardiovascular disease and atrial fibrillation) and the prescription of nephrotoxic drugs.

\section{METHODS \\ Data}

We used the Clinical Practice Research Datalink (CPRD) ${ }^{15}$ to construct an open cohort study of adults $(\geq 18$ years of age) registered at UK general practices whose data quality was deemed to be 'up-to-standard', that is, the data committed by general practices has reached a standard suitable for research (based on a CPRD algorithm that primarily focusses on death recording and gaps in the data).

\section{Study period}

We selected a start date of 1 April 2005, which postdated the publication of the K/DOQI guidelines for classification of CKD in 2002, ${ }^{2}$ and the introduction of QOF in UK primary care in $2004 .^{3}$ The study end date was 31 March 2013.

\section{Inclusion and exclusion criteria}

Eligible patients had to have been registered with their practice for a minimum of 12 months before their study entry to ensure adequate recording of baseline covariates. The study entry date was defined as the latest of either the study start date (1 April 2005) or the date of the patient's current registration date +12 months. We excluded patients who were living kidney donors, had a renal transplant, ever underwent dialysis or women who were pregnant in the 12 months prior to study entry. Follow-up ended at the study end date, unless preceded by the patient's death, transfer out of CPRD, the last available linked data, or (where applicable) pregnancy, renal transplantation/donation or dialysis. For any given financial year, patient records were excluded if their data were incomplete/censored.

\section{Outcomes}

A serum creatinine test was deemed to have occurred when a patient test record contained a valid date, an entity type associated with serum creatinine testing or blood/serum biochemistry and a Read code for serum creatinine testing (online supplementary table 1).

A proteinuria test was deemed to have occurred when a patient test record contained a valid date, an entity type associated with urine biochemistry tests and a Read code 
for albuminuria or proteinuria testing (online supplementary table 2).

A protocol-specified additional analysis, of Read codes for kidney function tests that could not be identified as serum creatinine or proteinuria, could not be carried out because use of these codes was highly heterogeneous by practice, with some practices making extensive use and other no use of such codes.

Subsequent tests recorded per patient on the same day were discarded, as these appeared to either be multiple abstractions from the same sample or data entry anomalies.

\section{Variables}

Nominal CKD stage was identified by Read codes (online supplementary table 1). Albuminuria status was derived using either ACR or protein:creatinine ratio (PCR). When these were unavailable, raw albumin excretion rate or protein excretion rate were used. Normoalbuminuria (albuminuria stage A1) was defined as $<3 \mathrm{mg} /$ mmol, microalbuminuria (albuminuria stage A2) was defined as $3-30 \mathrm{mg} / \mathrm{mmol}$ and macroalbuminuria (albuminuria stage A3) as $>30 \mathrm{mg} / \mathrm{mmol}$, in accordance with the 2012 KDIGO guidelines for evaluation and management of CKD. ${ }^{12}$ eGFR was calculated using the four-part Modification of Diet in Renal Disease (MDRD) equation based on recorded values of serum creatinine, sex, age at test and ethnicity. ${ }^{16}$ The four-part MDRD equation was used in place of the Chronic Kidney Disease Epidemiology Collaboration (CKD-EPI) equation, ${ }^{17}$ more recently advocated by NICE, as this was the equation that would predominantly have been used to monitor patients during the follow-up period.

Prevalence data for the comorbidities of: atrial fibrillation, cancer, diabetes, heart failure, hypertension, ischaemic heart disease, peripheral vascular disease, stroke or transient ischaemic attack and thyroid disease were assessed by the presence of diagnostic Read codes in patient clinical records.

Pharmacotherapies that were either nephrotoxic, excreted by the kidneys or that affected serum potassium were established through consensus between the general practitioners/pharmacologists (JA, CAO and CJT). These consisted of: ACE inhibitors, angiotensin receptor blockers, amiodarone/dronedarone, digoxin, diuretics, gold, immunosuppressants, lithium, mesalazine, non-steroidal anti-inflammatory drugs and oral anticoagulants (OACs).

Patient demographic data were also extracted, including age, gender, ethnicity, deprivation and region. Within these variables, age was categorised into seven levels (18-39, 40-49, 50-59, 60-69, 70-79, 80-89 and 90+ years), ethnicity was divided into six categories ('white', 'Asian', 'black', 'mixed', 'other' or 'missing'), deprivation was categorised into six levels (representing quintiles of IMD data plus a 'missing' level) and region was divided into 13 categories (aligning with the 10 SHAs of England and the countries of Northern Ireland, Scotland and Wales).

\section{Analyses}

Trends across kidney disease categorisations

Adherence to the most current NICE guidelines ${ }^{1}$ was evaluated by stratifying crude rates of serum creatinine and proteinuria testing (herein jointly referred to as 'kidney function testing') by CKD stage, and eGFR and albuminuria categories. We present these rates as tile and line plots.

\section{Trends over time}

We calculated crude rates of kidney function tests, stratified by financial year, and further stratified by CKD stage, demographic factors (such as age, ethnicity and deprivation), the presence of various comorbidities and concurrent prescriptions for nephrotoxic drugs. We present the percentage of patients with 1, 2, 3, 4 and $>4$ tests per financial year for serum creatinine and urinary albumin/ protein as bar plots.

\section{Factors associated with kidney function testing}

We examined factors associated with serum creatinine testing in CPRD. We used a mixed effects Poisson model implemented as a negative binomial regression $\operatorname{model}^{18}$ to assess the relationship between demographic factors, the presence of markers for CKD and other chronic conditions and indicators of drug therapy. We fitted age and year of entry into the study as categorical factors in order to model non-linear associations. The presence of Read codes for CKD was used as markers of kidney disease. We studied 13 geographic regions corresponding to the SHAs of England and the countries of Scotland, Northern Ireland and Wales. The model was adjusted for the presence of chronic conditions and medications. These were added to the models as binary covariates if a Read code or Gemscript code was present within the eligible data preceding the study entry date for that patient. The outcome of the model was the number of serum creatinine tests on record following study entry with the log personyears of follow-up used as the offset term. The model, therefore, estimates the natural log rates of serum creatinine testing, and covariate effects are $\log$ incidence rate ratios (IRRs). We have presented results from unadjusted, that is, univariable, minimally adjusted, that is, adjusted for gender, age, ethnicity, deprivation, region and year of entry and fully adjusted, that is, adjusted for all extracted variables, models on a natural scale, as IRRs with $95 \%$ CIs.

\section{Statistical software and packages}

All analyses were conducted in R (V.3.5.1) ${ }^{19}$ Plots were produced using the ggplot2 package. ${ }^{20}$ Crude rates and their 95\% CIs were calculated via the skewness-corrected asymptotic score method ${ }^{21}$ implemented in the scaspci() function of the ratesci package. ${ }^{22}$ Negative binomial models were fit using the glm.nb() function of the MASS package. $^{23}$

\section{Patient and public involvement}

This project has been reviewed by individuals with longterm conditions that require frequent monitoring, as 
well as nurse practitioners and general practice commissioners. Patient and public involvement members have also been invited to the steering and senior management groups. A patient and public involvement expert was also involved as a strategic consultant in a programme of work including this project.

\section{RESULTS}

\section{Baseline demographics}

We identified 4573275 patients from 630 practices with 26496643 person-years of eligible follow-up data, containing a total of 15049063 serum creatinine tests and 995524 proteinuria tests. The median length of follow-up was 6.1 (IQR 3.5-9.0) years. The cohort comprised 49.7\% men and $50.3 \%$ women. The median (IQR) age was 46 (34-61) years (table 1).

\section{Trends across kidney disease categorisations CKD categories}

When categorising kidney disease according to CKD stages, the highest rates of kidney function testing were found in patients with CKD stage 4 (figure 1). The lowest rates of testing were observed in patients without a Read code for CKD; however, such patients were still receiving roughly one serum creatinine test every 2 years and one proteinuria test every 9 years. Rates of serum creatinine testing were roughly in line with NICE guidelines for CKD stages $1-4$ but fell below recommendation in stage $5 .{ }^{1}$

\section{Estimated glomerular filtration rate and albuminuria categories}

The rates of serum creatinine testing observed in the data were higher than those advocated by NICE ${ }^{1}$ and $\mathrm{KDIGO}^{24}$ in all eGFR-albuminuria subcategories (figure 2). The highest rates of serum creatinine testing were in patients with eGFR stage G5. Patterns of proteinuria testing were less consistent, with patients with eGFR stage G5 or albuminuria stage A3 both exhibiting high rates of proteinuria testing. Rates of kidney function testing were generally higher than expected for individuals with either no eGFR or proteinuria stage assigned. For instance, patients with no assignable eGFR category and normal (A1) albuminuria levels were still receiving 0.84 (95\% CI 0.84 to $0.85)$ serum creatinine tests per year, while patients with no assignable albuminuria level and normal (G1) eGFR levels were still receiving 0.25 (95\% CI 0.25 to 0.25 ) proteinuria tests per year.

\section{Trends over time}

\section{Trends across CKD categories}

Figure 3 shows trends in serum creatinine (left panel) and urinary protein testing (right panel), stratified by stage of CKD for the same period. Rates of kidney function testing increased with CKD stage up to stage 4, though rates in CKD stage 5 were lower or similar to rates in CKD stage 4 . For patients in stages 2-5, rapid increases in the number of serum creatinine tests coincided with the inclusion of CKD management in QOF in 2006-2007 $7^{4}$ and then stabilised after 2007-2008. Sharp increases in proteinuria testing for patients with CKD stages 2-5 also coincided with the incorporation of proteinuria testing into QOF guidelines for the monitoring of CKD in 2009-2010. ${ }^{6}$

\section{General trends in testing}

The percentage of patients receiving kidney function tests has been steadily increasing year on year (figure 4). In the 2005-2006 financial year, $27.2 \%$ of patients received a serum creatinine test, while $7.5 \%$ of patients received a proteinuria test. In the 2012-2013 financial year, these figures were $38.1 \%$ and $11.8 \%$, respectively. These increases appear to be driven by increases in the number of patients with isolated kidney function testing, that is, patients receiving one test per year, which for serum creatinine tests increased from $18.5 \%$ in $2005-2006$ to $25.2 \%$ in 2012-2013. For proteinuria testing, isolated testing increased from $5.6 \%$ in $2005-2006$ to $9.1 \%$ in 2012-2013. In the same time period, the percentage of patients with repeated serum creatinine testing, that is, two or more tests per year, increased from $8.7 \%$ to $12.9 \%$, while the percentage of patients with repeated proteinuria testing increased from $2.0 \%$ to $2.7 \%$.

\section{Trends in testing across demographic data}

figure 5 shows the yearly trend in testing for serum creatinine (left panel) stratified by age and the equivalent trends in urinary protein tests (right panel). In general, rates of testing were higher with higher age, up to age $80-89$ years, but note that rates in the $90+$ years age group are not the highest. Serum creatinine test rates increased approximately linearly over time within each decile of age. In contrast, urinary protein test rates were constant over time in age groups less than 60 years and increased over time for patients over 60 years of age, with a sharp increase in the year 2009-2010.

Differences between the rates of kidney function testing were much lower when stratifying by gender (online supplementary figure 1), ethnicity (online supplementary figure 2), IMD quintile (online supplementary figure 3) and geographic region (online supplementary figure 4). Testing was marginally higher in women than men for both serum creatinine and proteinuria tests, with rate differences of roughly 0.1 tests per year and 0.02 tests per year, respectively. These differences remained relatively constant throughout the follow-up period. Testing remained higher in patients coded in the CPRD as white or mixed ethnicity, with patients of black or Asian ethnicity having lower rates of testing. A similar pattern was found in proteinuria testing. Rates of kidney function testing were similar when stratifying by IMD quintile, with rates being lowest in the lowest (most affluent) IMD quintile, for both markers of kidney function. Stratification by SHA region resulted in slightly larger differences in testing rates of up to 0.25 tests per year for serum creatinine and 0.14 tests per year for proteinuria. London demonstrated the lowest rates of kidney function testing for the majority of the study observation period. The 
Table 1 Characteristics of the cohort at study entry (note: missing categories omitted)

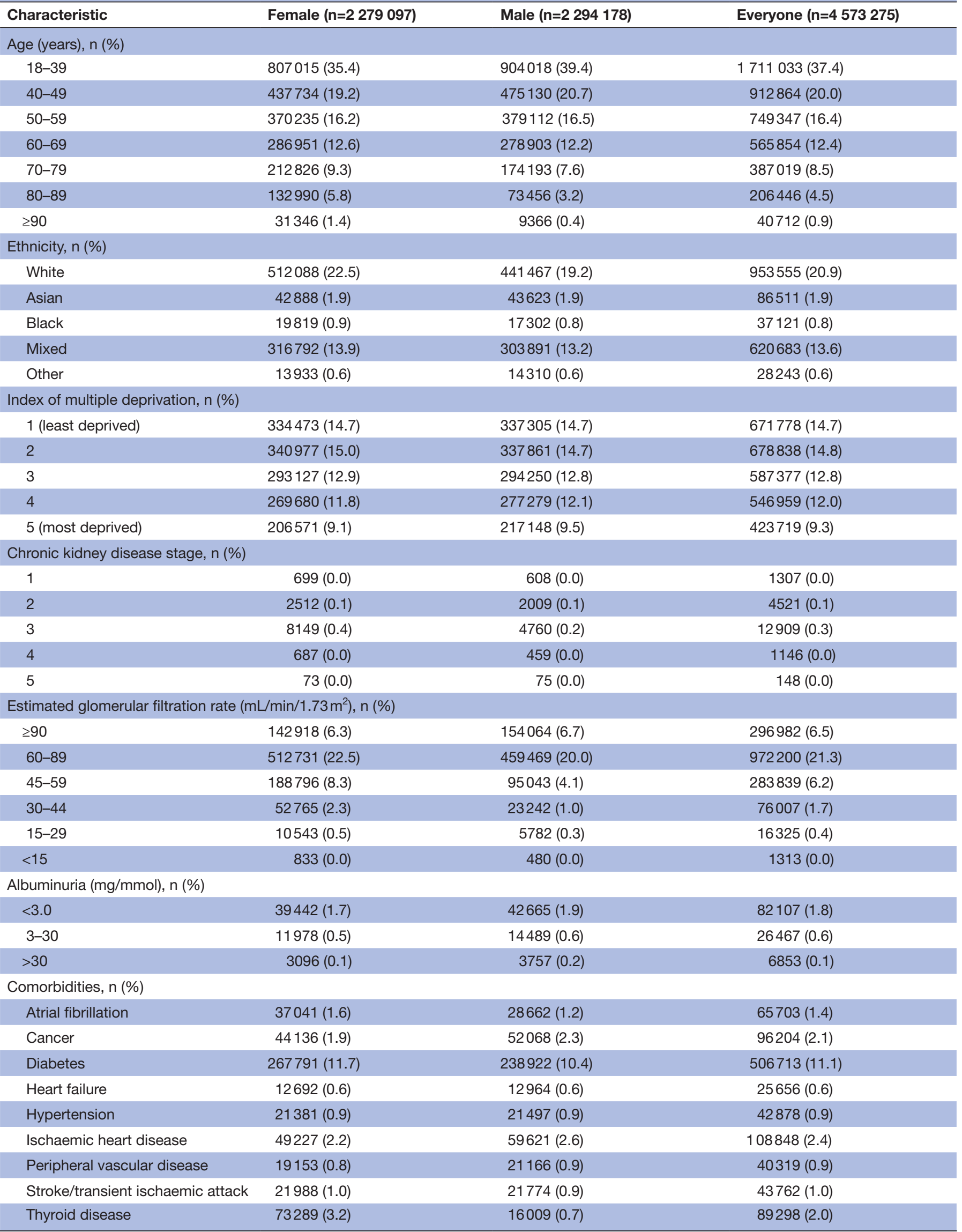




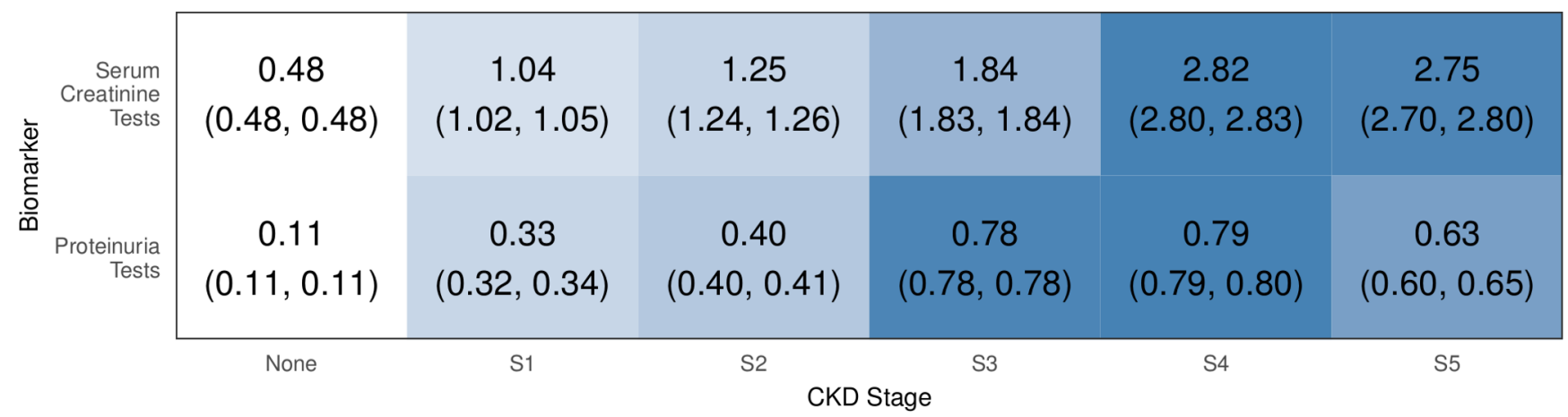

Figure 1 Rates of kidney function testing (per year), stratified by CKD stage. CKD, chronic kidney disease.

highest rates of serum creatinine testing were initially seen in North East England, being surpassed by Northern Ireland in 2007-2008. Rates of serum creatinine testing were initially lowest in Scotland and London, until 2010-2011, where rates of testing in Scotland increased. Conversely, the highest rates of proteinuria testing were present in the English East Midlands.

Trends in testing across comorbidities and pharmacotherapies

For all evaluated comorbidities, rates of kidney function testing were elevated when compared with a population for whom these comorbidities were absent (figure 6). Testing appears to have increased across all comorbidities with time, with the exception of diabetes, where the rate of testing appears to have decreased. The highest rates of serum creatinine testing were present in patients with heart failure and diabetes; however, all comorbidities were associated with at least one serum creatinine test per year by 2007-2008. The highest rates of proteinuria testing were present in patients with diabetes.

Across all evaluated pharmacotherapies, rates of kidney function testing were higher than in patients for whom prescriptions of these therapies were absent (figure 7). Rates of kidney function testing were relatively stable across time for most comorbidities with a few notable exceptions. For patients receiving prescriptions for gold, methotrexate or other immunosuppressants, serum creatinine testing appears to have increased with time. Proteinuria testing was elevated in patients prescribed

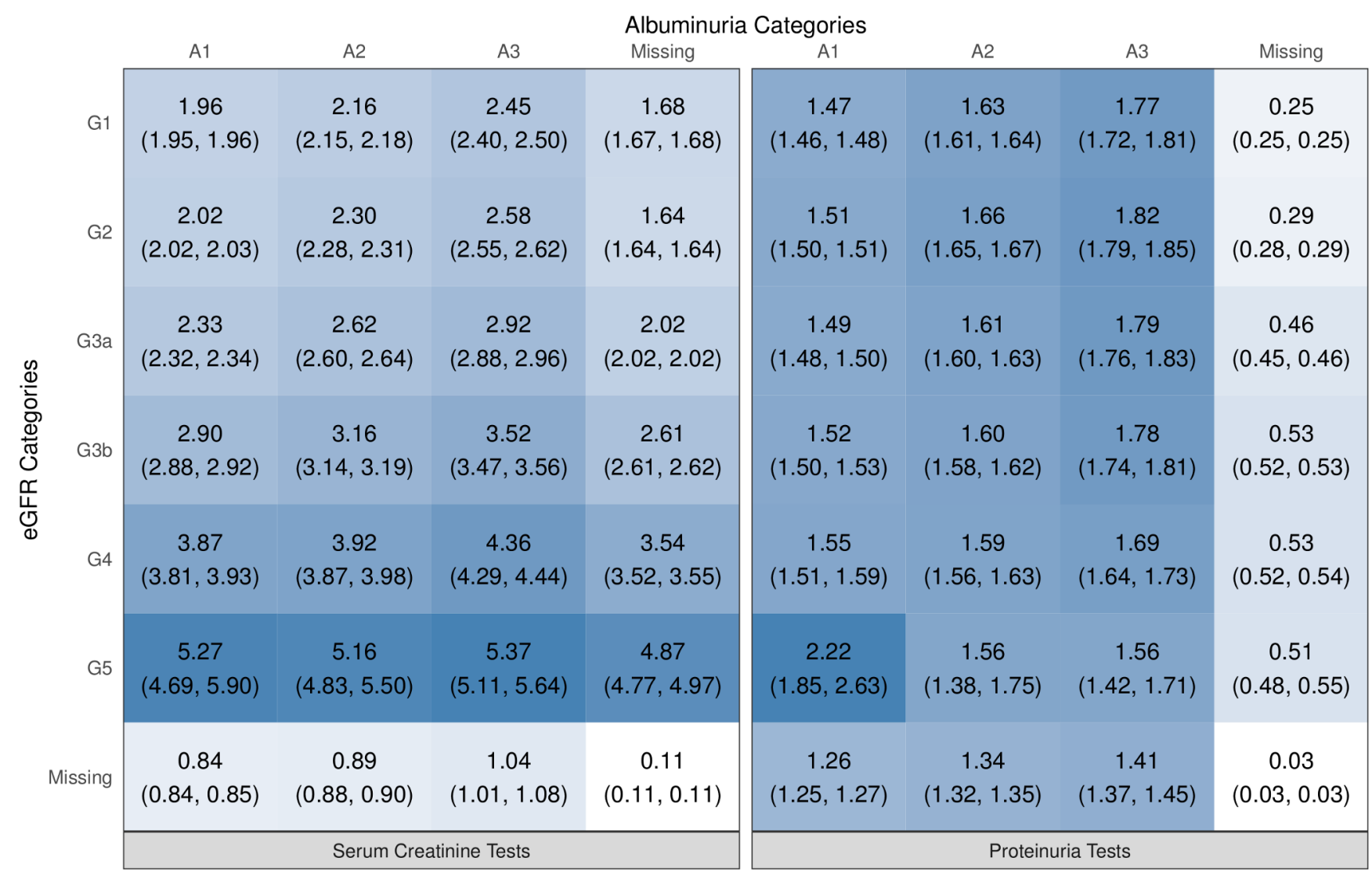

Figure 2 Rates of kidney function testing (per year), stratified by eGFR and albuminuria categories. eGFR, estimated glomerular filtration rate. 


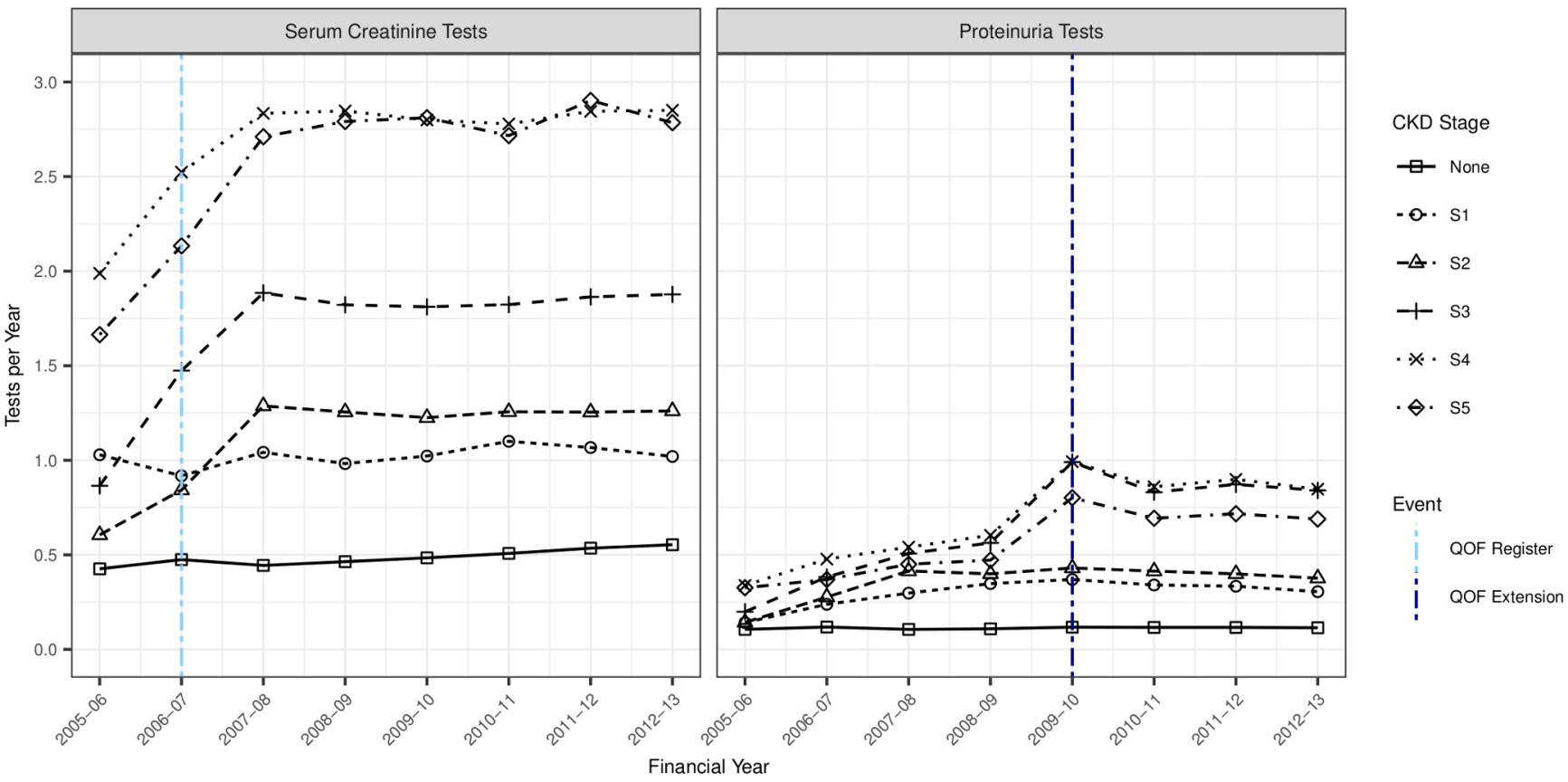

Figure 3 Rates of kidney function testing per financial year, stratified by CKD stage. CKD, chronic kidney disease; QOF, Quality and Outcomes Framework.

gold but was generally less than 0.5 tests/year for all other pharmacotherapies.

\section{Factors associated with serum creatinine testing}

The presence of a Read code for CKD was independently associated with more frequent serum creatinine testing in primary care, with stage 4 CKD conferring the highest rates of testing (table 2). Testing frequency increased with age up to a peak at ages $80-89$ years. Variation in testing between the
SHA regions of England was quite low, with the exception of the North East and the South West, where the rates of testing were roughly $20 \%$ higher than that of London. Rates in Northern Ireland, Scotland and Wales were $21 \%-48 \%$ greater than those of London, possibly reflecting differences in clinical guidelines between England and other countries. In our adjusted model of testing frequency, the extent of testing in men and women differed by 14\% IRR 1.14

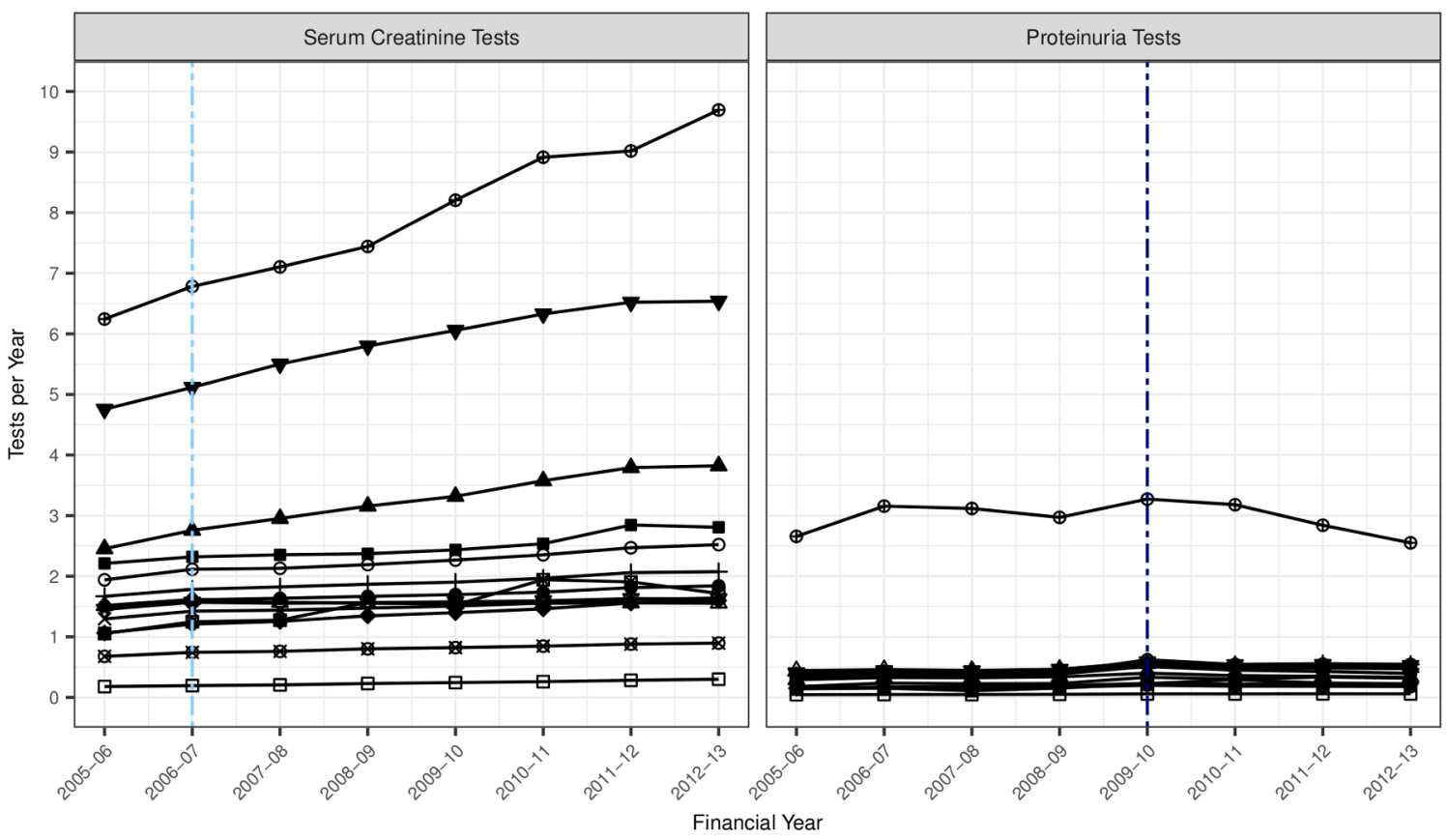

Pharmacotherapy

七- None

$\simeq \mathrm{ACE}-\mathrm{is}$

$\nabla$ ARBs

$\neg$ Darones

+ Digoxin

$\rightarrow$ Diuretics

\&-Ethambutol

- Gold

$\sim$ Immuno

$\rightarrow$ Lithium

$\neg$ Mesalazine

$\checkmark$ Methotrexate

NSAIDS

$\multimap$ OACs

Event

QOF Register

I QOF Extension

year. NSAIDs, non-steroidal anti-inflammatory drugs; OACs, oral anticoagulants. 


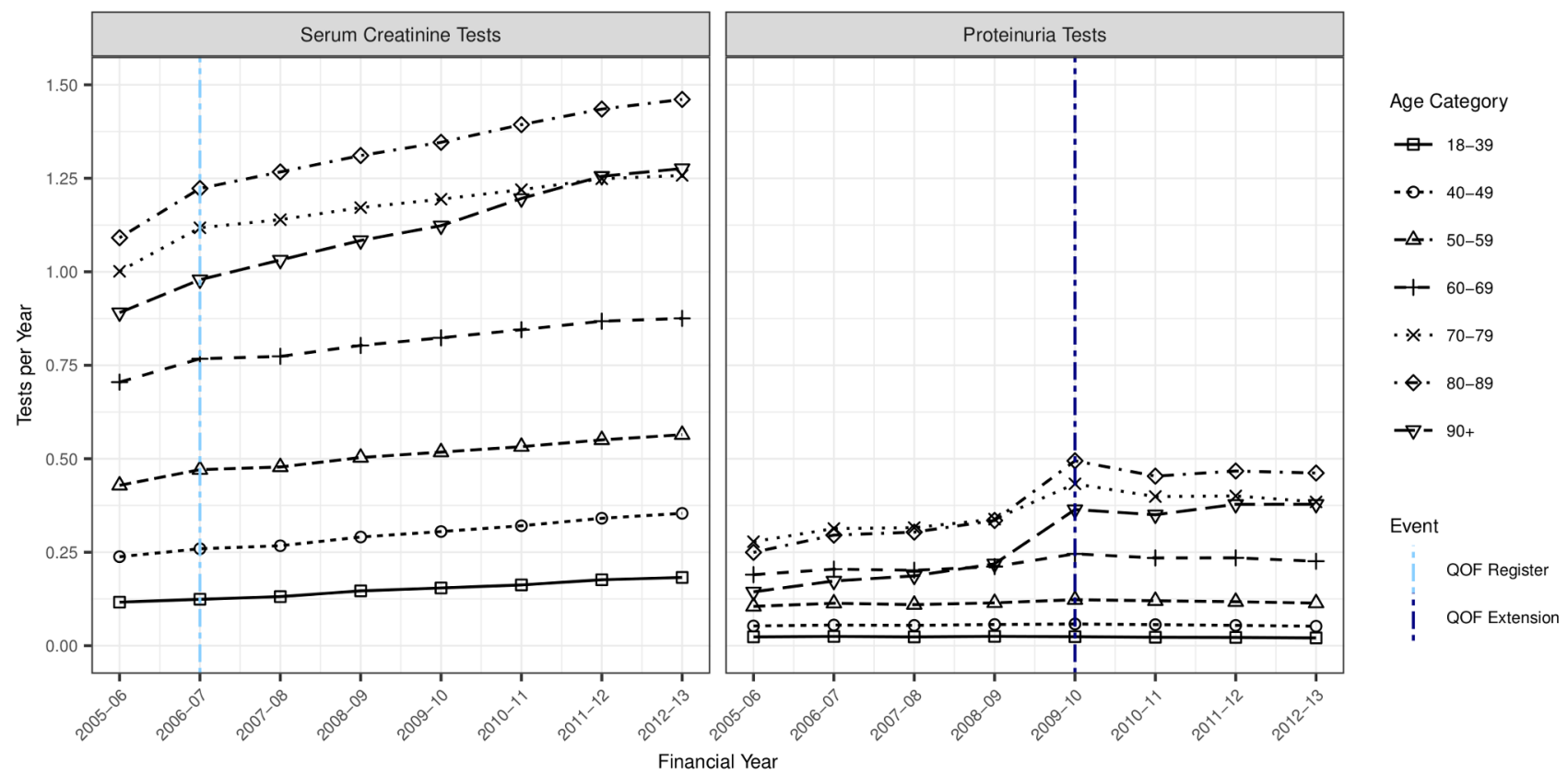

Figure 5 Rates of kidney function testing per financial year, stratified by age category. QOF, Quality and Outcomes Framework.

(95\% CI 1.14 to 1.14). All assessed comorbidities were significantly associated with elevated rates of serum creatinine testing with the exception of atrial fibrillation. With the exception of ethambutol, for all analysed pharmacotherapies, serum creatinine testing increased independently of other factors and was most marked in patients taking methotrexate, other immunosuppressants, gold and lithium.

\section{DISCUSSION \\ Key results}

This is the first study to evaluate the rates of kidney function testing over a 9-year period following the introduction of the QOF in a large UK primary care database. Over the course of this study, rates of serum creatinine and proteinuria testing increased by $40 \%$ and $36 \%$, respectively, and by 2012-2013 almost 4 in every 10 people were
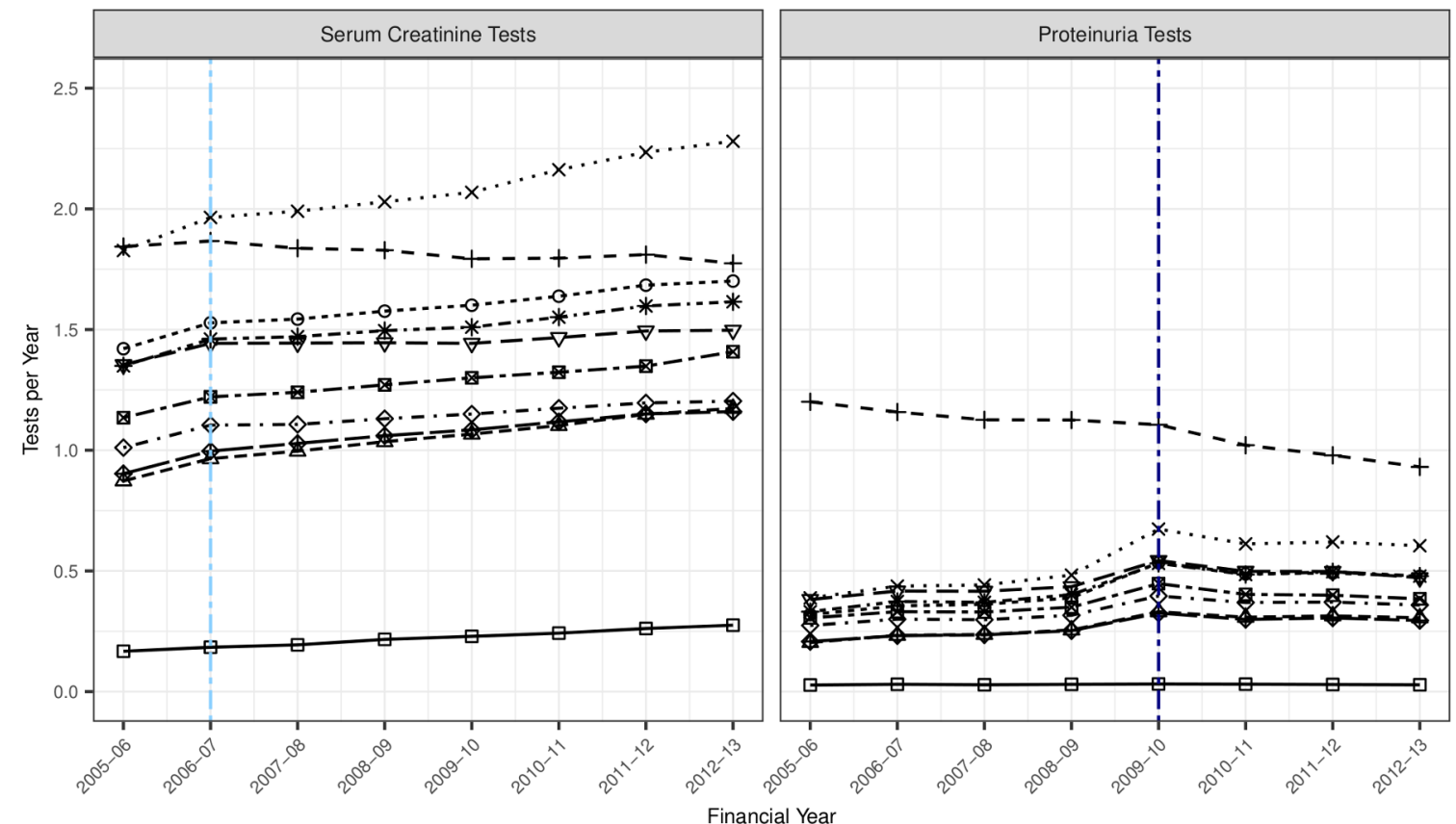

Comorbidity
$\square$ - None
- - AFib
$-A \cdot$ Cancer
+ Diabetes
$\cdot \rtimes \cdot$ HF
$\cdot \diamond \cdot$ HTN
$\nabla-$ IHD
$-母 \cdot$ PVD
$\cdot *$. Stroke/TIA
- THY Disease
Event

Figure 6 Rates of kidney function testing per financial year, stratified by comorbidity. AFib, atrial fibrillation; HF, eart failure; HTN, hypertension; IHD, ischaemic heart disease; PVD, peripheral vascular disease; QOF, Quality and Outcomes Framework; TIA, transient ischaemic attack; THY, thyroid. 

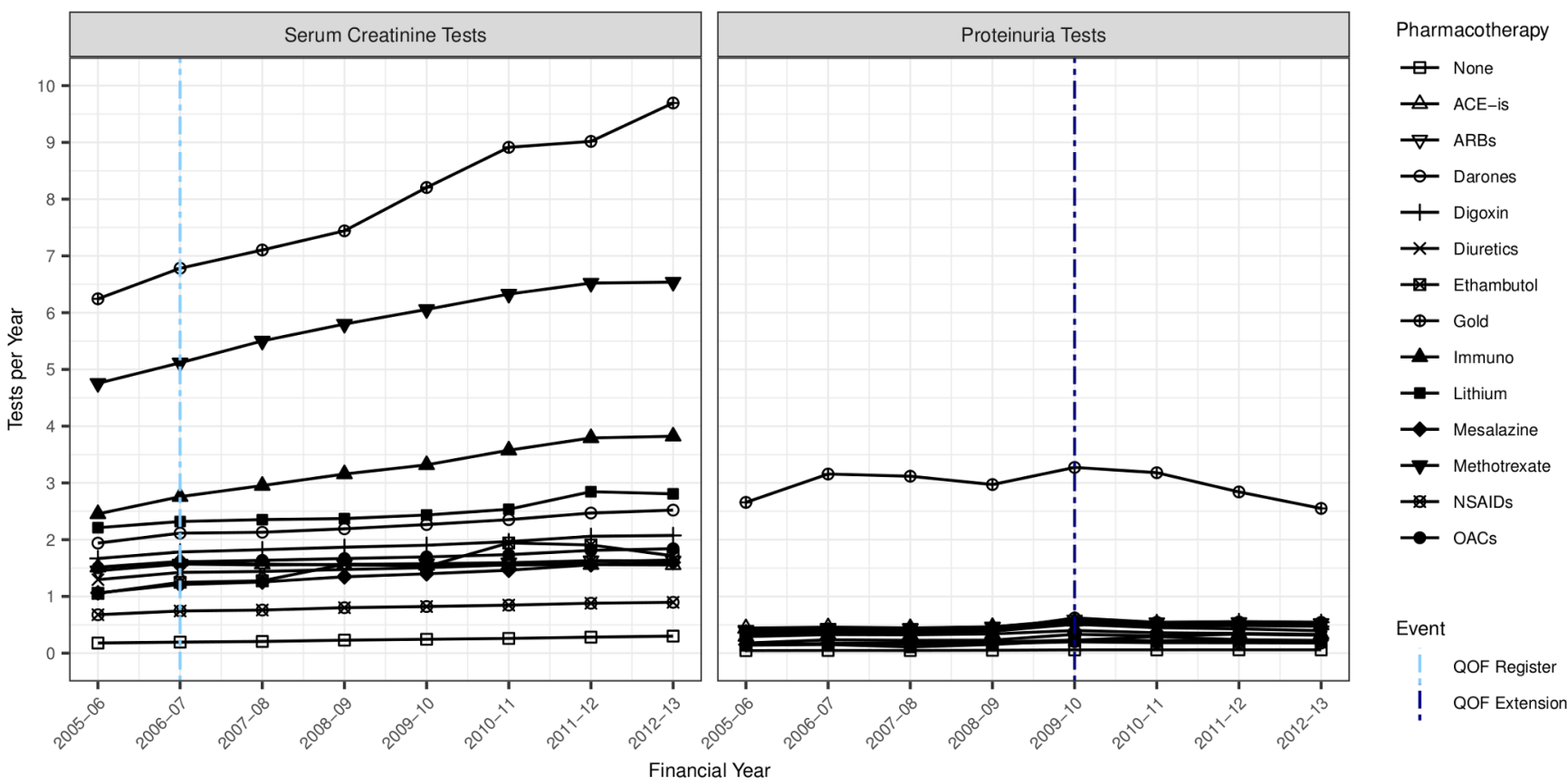

Figure 7 Rates of kidney function testing per financial year, stratified by concomitant pharmacotherapy. ACE-is, ACE inhibitors; ARBs, angiotensin II receptor blockers; Darones, amiodarone or dronedarone; Immuno, other (non-methotrexate) immunosuppressants; NSAIDs, non-steroidal anti-inflammatory drugs; OACs, oral anticoagulants; QOF, Quality and Outcomes Framework.

receiving at least one serum creatinine test per year and over one in every 10 people were receiving at least one proteinuria test per year.

Across most strata evaluated, rates of kidney function testing appear to have either remained constant or increased with time. One notable exception to this was diabetes, where rates appear to have decreased with time. Women appear to be tested more than men, receiving roughly an extra 0.1 serum creatinine tests per year and an extra 0.02 proteinuria tests per year. This may be because women are more likely to schedule and attend appointments in primary care, as evidenced by a UK national study of patient factors associated with missed appointments. ${ }^{25}$ When stratifying by age, rates of kidney function testing increased between successive age categories up to age $80-89$ years, with patients aged $\geq 90$ years typically having lower rates of testing than patients aged 70-79 years. Serum creatinine and urinary protein testing have both increased across all ethnic groups, but patients of white and mixed ethnicities still have higher rates of testing than patients of black and Asian ethnicity. Stratification by IMD quintile demonstrated minimal differences in testing rates. Conversely, stratification by comorbidity revealed the highest rates of both serum creatinine and proteinuria testing to be present in patients with heart failure or diabetes. Creatinine testing is undertaken in the primary care practice in the UK, rather than in a separate facility, and therefore, creatinine testing is sensitive to factors that influence practice attendance. However, some patients with diabetes will be managed by specialists as part of an outpatient hospital service and will have blood tests requested and taken at the hospital. These blood tests will not be sent to primary care electronic health records and will not appear in CPRD. The smaller rate of testing seen in this database for patients with diabetes may therefore not reflect deficiencies in overall care, but simply the fact that care is shared with the hospital for some of those patients. Stratification by concomitant pharmacotherapy revealed the highest rates of kidney function testing to be present in patients prescribed gold. Serum creatinine testing was also more frequent in patients prescribed immunosuppressants.

The effects of pay-for-performance indicators are visible in most plots present in this paper with noticeable increases in the rates of serum creatinine testing in 2006-2007 and of proteinuria testing in 2009-2010. The former of these coincided with the requirement that general practices maintain a register of patients with CKD stages $3-5,{ }^{4}$ while the latter coincided with the inclusion of the monitoring of secondary markers of kidney disease via ACR and PCR tests in patients on the CKD register. ${ }^{5}$ There was no obvious impact in any of the plots from the 2008-2009 NICE guidelines that recommended monitoring eGFR levels in high-risk patients. ${ }^{5}$

Frequency of serum creatinine testing was strongly associated with increasing age and the presence of a Read code for CKD in adjusted analyses. Testing frequency was also independently associated with chronic conditions and prescription of potentially nephrotoxic drugs but has risen year on year, even after accounting for age, chronic conditions and prescription of drugs that require monitoring of kidney function. 
Table 2 Results of regression models describing the demographic characteristics, the presence/absence of chronic conditions and drug prescription and associations with the frequency of serum creatinine testing in primary care

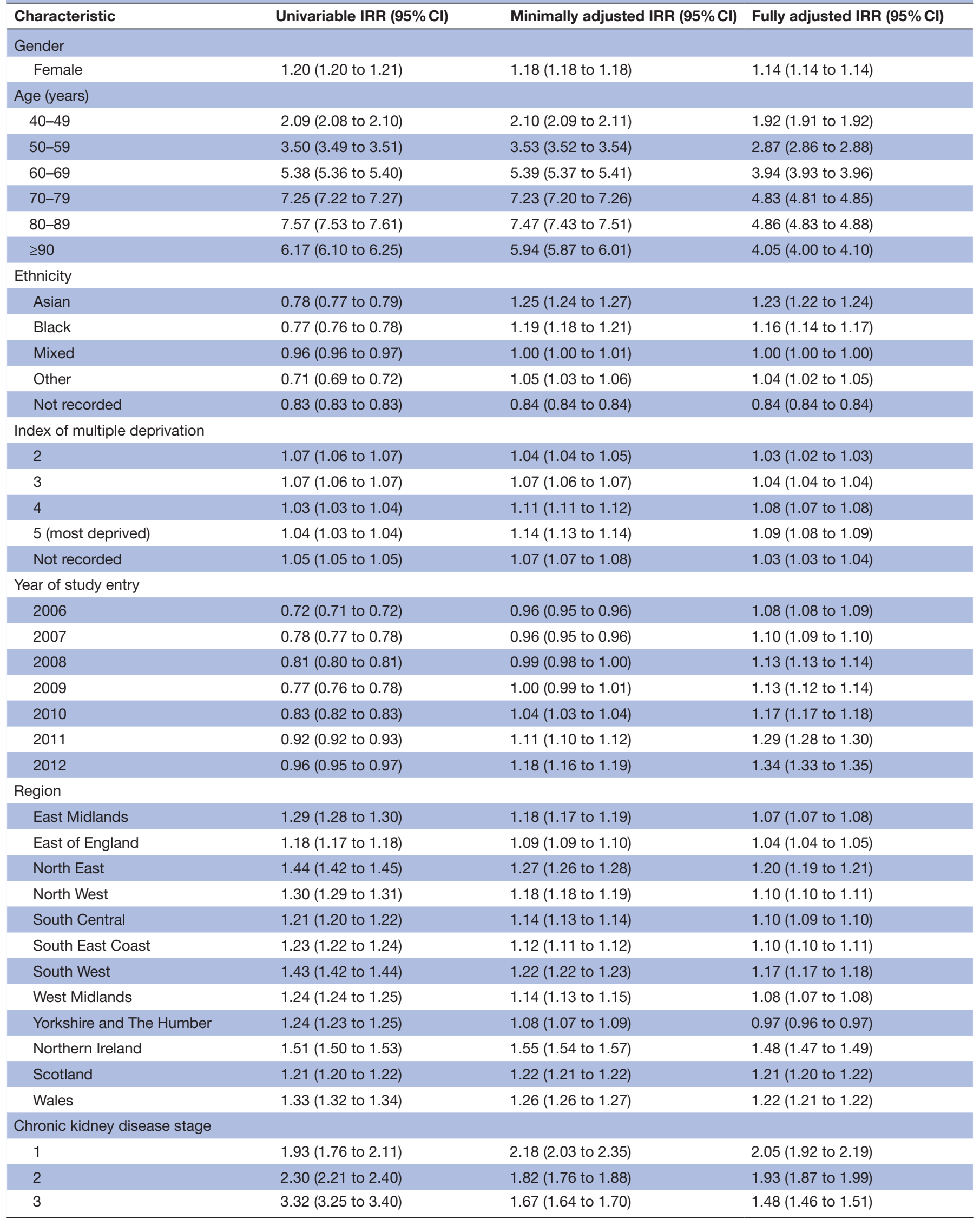


Table 2 Continued

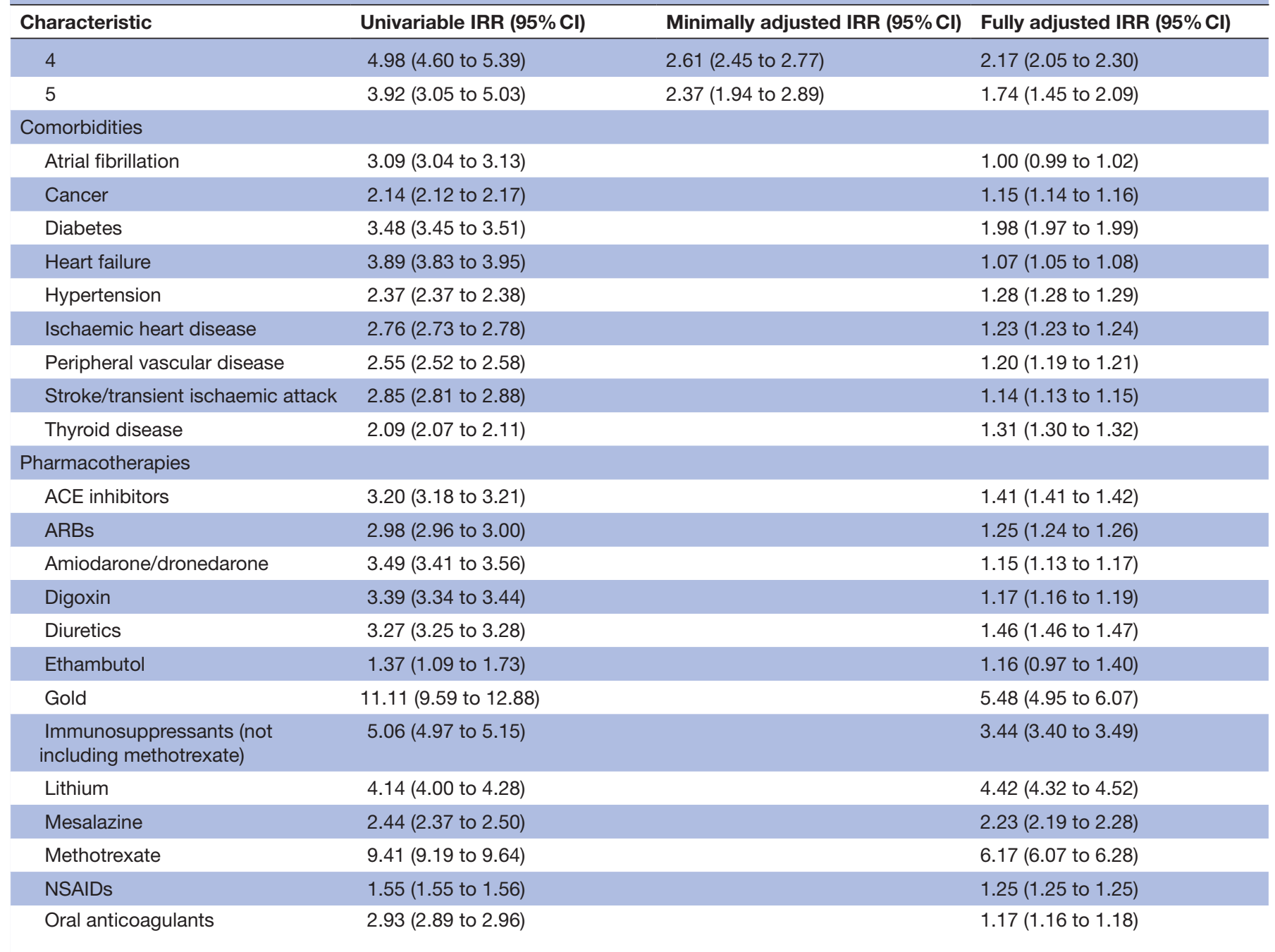

IRR, incidence rate ratio; NSAIDs, non-steroidal anti-inflammatory drugs.

\section{Strengths and limitations}

To date, this is the largest population-based study of trends in renal function testing in primary care. The study population was an unselected sample of over 4.5 million patients from over 600 general practices across the UK included in the CPRD database, which has been shown to be representative of the UK. The scale and design of the study allowed us to test associations adjusted for many important potential explanatory and confounding factors. Our study has limitations, some of which are inherent in the CPRD database. We were not able to ascertain why the tests were performed. Even though the CPRD contains consultation codes, these provide only a very broad classification of the time and type of consultation (eg, clinic, night visit and home visit). An in-depth analysis of Read codes or mining of the consultation free text would be required to start to explain the reasons for test ordering, which is beyond the scope of this study. Finally, the use of the MDRD equation could be challenged. It was the formula in use during the period of the study but is now considered inferior to the CKD-EPI formula. ${ }^{17}$ However, we have used MDRD, because we wanted the analysis to reflect the clinical decision made at the time of the study.

\section{Relationship to the literature}

The rise in the number of patients having serum creatinine concentration measurements and the increased frequency of testing for those being tested can be interpreted in two ways. CKD has gained more attention since the incorporation of CKD into the QOF in 2006-2007. The establishment of a register in 2006-2007 and its subsequent extension has encouraged renal function testing to identify those with CKD who may benefit from risk factor modification. From the viewpoint of patient safety, our results are encouraging and show that, for all the therapies we examined, the prescription of drugs that are potentially nephrotoxic is associated with more frequent monitoring.

Our results could be interpreted in a different light. There is little direct evidence that extra testing has 
improved outcomes in the short term or long term. ${ }^{26}$ Additional testing has increased the apparent prevalence of CKD from $0.12 \%$ to $6.51 \%$, but as yet, there has been no change in long-term outcomes, such as patients requiring renal replacement therapy. ${ }^{27} 28$ Increases in consultations with general practitioners or practice nurses for either newly diagnosed disease or monitoring, with associated laboratory tests, place further strain on limited healthcare resources and increase expenditure. The very substantial costs of renal replacement therapy or cardiovascular complications ${ }^{28}$ mean that testing might be cost-effective, even if it results in only modest reductions in the number of patients who progress to this stage, but whether this is the case is unclear. In a report from one NHS trust in the period following the introduction of renal QOF, there was an abrupt $61 \%$ increase in the number of new referrals to nephrology, $54 \%$ of which were classified as inappropriate and a further $22 \%$ as inadequate. ${ }^{29}$ Inappropriate referrals use up resources and may cause unnecessary distress to patients and their carers. ${ }^{30}$

\section{Implications for practice}

Rates of testing have increased over the observation period in our study. Much of these increases appear to be driven by financial incentivisation schemes, such as the QOF. However, the increases were found to be independent of comorbidities, age and prescriptions for 'high risk' drugs. Much of the increase in testing appears to have occurred in patients with mildly to moderately impaired kidney function (CKD stages 2-3). However, there is limited evidence to suggest any benefit from interventions delivered in the early stages of CKD. ${ }^{31}$ Moreover, studies in cholesterol monitoring have shown that more frequent testing can have negative consequences, ${ }^{32}$ particularly for biomarkers that have high within-person variability, such as serum creatinine, ${ }^{33}$ with an increased likelihood of raising false alarms for increased CKD severity. Hence, a more targeted approach could prove beneficial for most patients.

Increases in testing are also likely to have knock-on effects to other aspects of healthcare, including the financial burden on the NHS, the time burden on general practitioners and laboratory workloads, potentially resulting in delayed or missed diagnosis. ${ }^{34}$ Reducing the amount of serum creatinine testing performed as part of kidney function monitoring could ease some of these burdens, although we acknowledge that a reasonable amount of serum creatinine testing is performed as part of test batches not directly related to the assessment of kidney function and including other tests such as full blood counts. ${ }^{35}$

\section{CONCLUSION}

The observed increase in kidney function testing could be attributable to any or all of several changes that have occurred over the period of the study. The introduction of pay-for-performance indicators, the establishment of a CKD register, national guidelines promoting monitoring of renal function in high-risk groups and linkage of pathology laboratories to practice systems have potentially all raised the profile of CKD in primary care and contributed to the observed increases in testing. While it is clear that these initiatives have changed process measures, it is still not clear whether clinical outcomes have improved as a consequence.

\section{Author affiliations}

${ }^{1}$ Nuffield Department of Primary Care Health Sciences, University of Oxford, Oxford, Oxfordshire, UK

${ }^{2}$ Institute of Applied Health Research, University of Birmingham, Birmingham, West Midlands, UK

${ }^{3}$ Nuffield Department of Medicine, University of Oxford, Oxford, Oxfordshire, UK

${ }^{4}$ John Radcliffe Hospital, Oxford Radcliffe Hospitals NHS Trust, Oxford, Oxfordshire, UK

${ }^{5}$ Bristol-Myers Squibb Pharmaceuticals Ltd, Uxbridge, Hillingdon, UK

Acknowledgements We would like to thank Alice Fuller and Dr Sarah Lay-Flurrie for their hard work in providing much of the initial data management for this project.

Contributors RP and RS provided substantial contributions to the concept and design of the study. JO, BF, RS, RP and EM provided statistical expertise, while JA, CT, COC and DL lent clinical and pharmacological expertise. Any coding work necessary for the project was performed by BF, JO, EM and NH. All authors contributed to the drafting and critical appraisal of the manuscript. Final approval for the version to be published was given by RP.

Funding This article presents independent research funded by the National Institute for Health Research (NIHR) under the programme grants for applied research programme (RP-PG-1210-12003). CT is funded through an NIHR academic clinical lectureship. RP receives funding from the NIHR Oxford Biomedical Research Centre Program, the NIHR Program for Applied Research, the NIHR Health Protection Research Unit Gastrointestinal Infections Group and the NIHR Diagnostic Evidence Co-operative.

Disclaimer The views expressed are those of the authors and not necessarily those of the NHS, the NIHR or the Department of Health and Social Care.

Competing interests NRH is currently employed by Bristol-Meyers Squibb Limited, a company that manufactures ACE inhibitors, which are drugs indicated in the treatment of chronic kidney disease, when present in conjunction with other comorbidities such as type 2 diabetes. CT reports speaker fees from Vifor and Novartis and non-financial support from Roche outside of the submitted work. All other authors declare no conflicts of interest.

\section{Patient consent for publication Not required.}

Ethics approval The protocol for this research was approved by the Independent Scientific Advisory Committee (ISAC) of the Medicines and Healthcare Products Regulatory Agency (protocol number 14_150R), and the approved protocol was made available to the journal and reviewers during peer review. Ethical approval for observational research using the Clinical Practice Research Datalink (CPRD) with approval from ISAC has been granted by a National Research Ethics Service committee (Trent Multi Research Ethics Committee, REC reference number 05/ MRE04/87)

Provenance and peer review Not commissioned; externally peer reviewed.

Data sharing statement The data that support the findings of this study are available from the Medicines and Healthcare Products Regulatory Agency, but restrictions apply to the availability of these data, which were used under licence for the current study and so are not publicly available. Data are, however, available from the Medicines and Healthcare Products Regulatory Agency, subject to approval from ISAC.

Open access This is an open access article distributed in accordance with the Creative Commons Attribution Non Commercial (CC BY-NC 4.0) license, which permits others to distribute, remix, adapt, build upon this work non-commercially, and license their derivative works on different terms, provided the original work is properly cited, appropriate credit is given, any changes made indicated, and the use is non-commercial. See: http://creativecommons.org/licenses/by-nc/4.0/. 


\section{REFERENCES}

1. National Institute for Health and Care Excellence, "Chronic kidney disease in adults: assessment and management. Clinical guideline [CG182]. 2014. https://www.nice.org.uk/guidance/cg182.

2. National Kidney Foundation. K/DOQI clinical practice guidelines for chronic kidney disease: evaluation, classification, and stratification. Am J Kidney Dis 2002;39(2 Suppl 1):S1-S266.

3. Department of Health, The National Services Framework for Renal Services. Part One: Dialysis and Transplantation, 2004.

4. British Medical Association. "Revisions to the GMS contract 2006/07. 2006 http://www.nhsemployers.org/-/media/Employers/ Documents/Primary-care-contracts/QOF/2006-07/Revisions-to-theGMS-contract-200607-Delivering-investments-in-general-practice. pdf?la=en\&hash=C4949A6E6518C75287E55BCB13A8D311 ADODF3E4.

5. National Institute for Health and Care Excellence. NICE Clinical Guideline 73: Chronic Kidney Disease: Early Identification and Management of Chronic Kidney Disease in Adults in Primary and Secondary Care. 2008 https://www.nice.org.uk/guidance/CG73.

6. British Medical Association, "Quality and Outcomes Framework guidance for GMS contract 2009/10”. 2009 http://www. nhsemployers.org/ /media/Employers/Documents/Primary care contracts/QOF/2010-11/Quality and Outcomes Framework guidance for GMS contract 200910 - Delivering investment in general practice. pdf.

7. McGovern AP, Rusholme B, Jones S, et al. Association of chronic kidney disease (CKD) and failure to monitor renal function with adverse outcomes in people with diabetes: a primary care cohort study. BMC Nephrol 2013;14:198.

8. de Lusignan S, Nitsch D, Belsey J, et al. Disparities in testing for renal function in UK primary care: cross-sectional study. Fam Pract 2011;28:638-46.

9. Doll H, Shine B, Kay J, et al. The rise of cholesterol testing: how much is unnecessary. Br J Gen Pract 2011;61:e81-e88.

10. Oke J, Shine B, McFadden E, et al. Trends in serum creatinine testing in Oxfordshire, UK,1993-2013: a population-based cohort study. BMJ Open 2015;5:e009459.

11. Zhi M, Ding EL, Theisen-Toupal J, et al. The landscape of inappropriate laboratory testing: a 15-year meta-analysis. PLoS One 2013;8:e78962.

12. Kidney Disease: Improving Global Outcomes (KDIGO) CKD Work Group, "KDIGO 2012 Clinical Practice Guideline for the Evaluation and Management of Chronic Kidney Disease,". Kidney Int Suppl 2013;3:1-150.

13. Charles RF, Powe NR, Jaar BG, et al. Clinical testing patterns and cost implications of variation in the evaluation of CKD among US physicians. Am J Kidney Dis 2009;54:227-37.

14. Choi M, Montgomery E, Saffer T, et al. Chronic Kidney Disease Change Package: Population Health Strategies for Cardiovascular and Kidney Disease Risk Reduction, 2018.

15. Herrett E, Gallagher AM, Bhaskaran K, et al. Data resource profile: Clinical Practice Research Datalink (CPRD). Int J Epidemiol 2015;44:827-36.

16. Levey AS, Bosch JP, Lewis JB, et al. A more accurate method to estimate glomerular filtration rate from serum creatinine: a new prediction equation. Modification of Diet in Renal Disease Study Group. Ann Intern Med 1999;130:461-70.
17. Levey AS, Stevens LA, Schmid $\mathrm{CH}$, et al. A new equation to estimate glomerular filtration rate. Ann Intern Med 2009;150(9):604-12.

18. Kirkwood BR, Sterne JAC. 31.4 Random Effects (Multilevel) Models," in Essential Medical Statistics. 2nd ed. Malden, Massachusetts, USA: Wiley-Blackwell, 2003:361-4.

19. Core Team R. R: A Language and Environment for Statistical Computing. Vienna, Austria: R Foundation for Statistical Computing, 2018.

20. Wickham H. ggplot2: Elegant Graphics for Data Analysis. New York: Springer-Verlag, 2016.

21. Laud PJ. Equal-tailed confidence intervals for comparison of rates. Pharm Stat 2017;16:334-48.

22. Laud PJ. ratesci: Confidence Intervals for Comparisons of Binomial or Poisson Rates, 2018.

23. Venables WN, Ripley BD. Modern Applied Statistics with S, Fourth. New York: Springer, 2002.

24. KDIGO. KDIGO 2012 Clinical Practice Guideline for the Evaluation and Management of Chronic Kidney Disease. Kidney Int Supp/ 2012;3:1136-50.

25. Ellis DA, McQueenie R, McConnachie A, et al. Demographic and practice factors predicting repeated non-attendance in primary care: a national retrospective cohort analysis. Lancet Public Health 2017;2(12):e551-e559.

26. Inker LA, Astor BC, Fox CH, et al. KDOQI US commentary on the 2012 KDIGO clinical practice guideline for the evaluation and management of CKD. Am J Kidney Dis 2014;63:713-35.

27. Byrne C, et al. UK Renal Registry UK Renal Registry 19th Annual Report of the Renal Association. Nephron 2017;137(suppl1).

28. Kerr M, Bray B, Medcalf J, et al. Estimating the financial cost of chronic kidney disease to the NHS in England. Nephrol Dial Transplant 2012;27 Suppl 3(Suppl 3):iii73-iii80.

29. O'Callaghan CA, Shine B, Lasserson DS. Chronic kidney disease: a large-scale population-based study of the effects of introducing the CKD-EPI formula for eGFR reporting. BMJ Open 2011;1:e000308.

30. Glassock RJ, Winearls C. Screening for CKD with eGFR: doubts and dangers. Clin J Am Soc Nephrol 2008;3:1563-8.

31. Fink HA, Ishani A, Taylor BC, et al. Screening for, monitoring, and treatment of chronic kidney disease stages 1 to 3 : a systematic review for the U.S. Preventive Services Task Force and for an American College of Physicians Clinical Practice Guideline. Ann Intern Med 2012;156(8):570-81.

32. Glasziou PP, Irwig L, Heritier S, et al. Monitoring cholesterol levels: measurement error or true change? Ann Intern Med 2008;148(9):656-61.

33. Lamb EJ, Brettell EA, Cockwell P, et al. The eGFR-C study: accuracy of glomerular filtration rate (GFR) estimation using creatinine and cystatin $\mathrm{C}$ and albuminuria for monitoring disease progression in patients with stage 3 chronic kidney disease--prospective longitudinal study in a multiethnic population. BMC Nephrol 2014;15(no. 1):13.

34. Gandhi TK, Kachalia A, Thomas EJ, et al. Missed and delayed diagnoses in the ambulatory setting: a study of closed malpractice claims. Ann Intern Med 2006;145(7):488-96.

35. Fanshawe TR, Ordóñez-Mena JM, Turner PJ, et al. Frequencies and patterns of laboratory test requests from general practice: a service evaluation to inform point-of-care testing. J Clin Pathol 2018;71:1065-71. 
Correction: Trends in kidney function testing in UK primary care since the introduction of the quality and outcomes framework: a retrospective cohort study using CPRD

Feakins B, Oke J, McFadden E, et al. Trends in kidney function testing in UK primary care since the introduction of the quality and outcomes framework: a retrospective cohort study using CPRD. BMJ Open 2019;9:e028062. doi: 10.1136/bmjopen-2018-028062.

This article was previously published with an error in the figure.

In the publication, Figure 4 is incorrect, and is a duplicate of figure 7 . The correct Figure 4 is below:

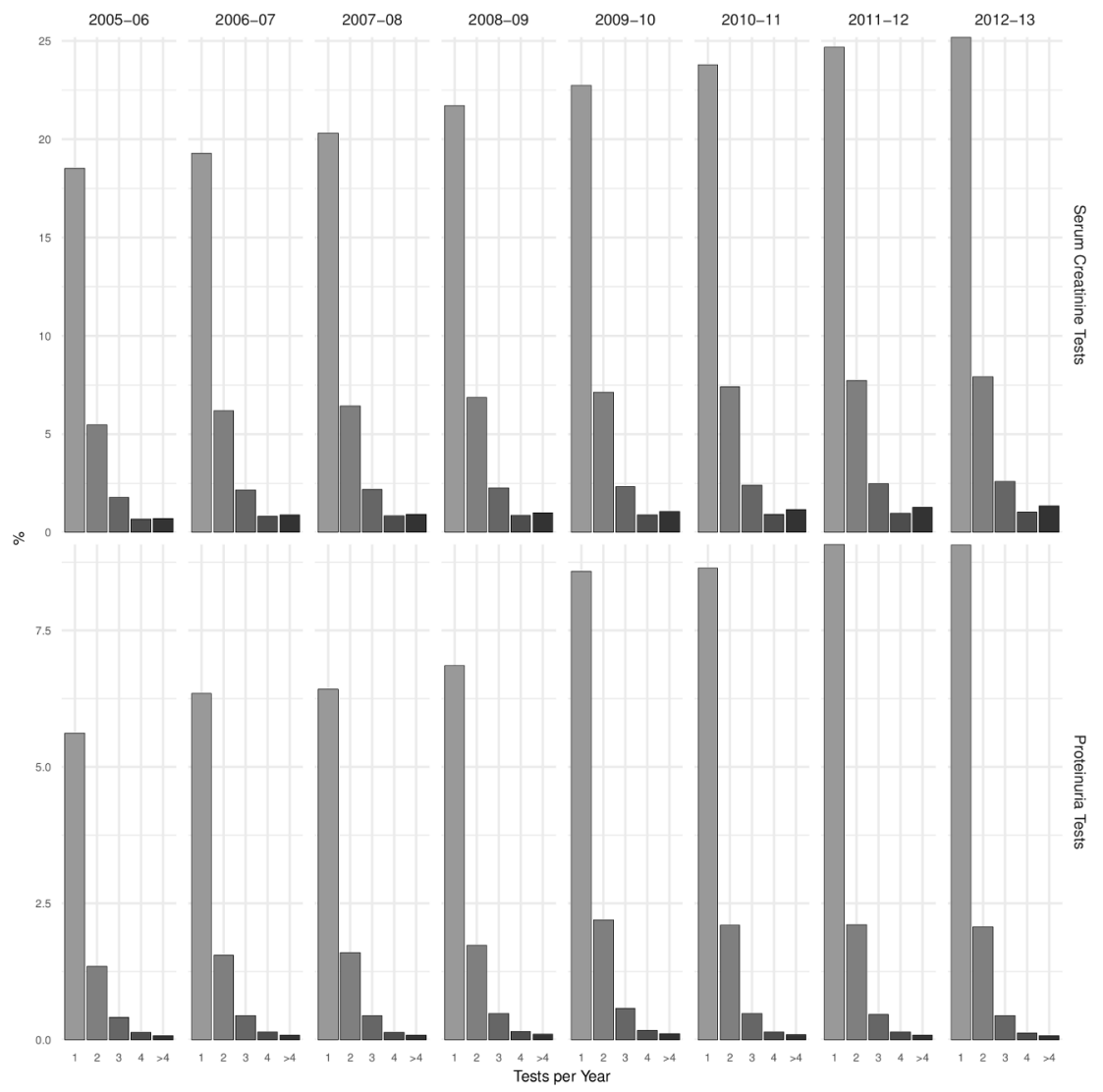

Open access This is an open access article distributed in accordance with the Creative Commons Attribution Non Commercial (CC BY-NC 4.0) license, which permits others to distribute, remix, adapt, build upon this work non-commercially, and license their derivative works on different terms, provided the original work is properly cited, appropriate credit is given, any changes made indicated, and the use is non-commercial. See: http://creativecommons.org/licenses/by-nc/4.0/.

(c) Author(s) (or their employer(s)) 2019. Re-use permitted under CC BY-NC. No commercial re-use. See rights and permissions. Published by BMJ.

BMJ Open 2019;9:e028062corr1. doi:10.1136/bmjopen-2018-028062corr1

A) Check for updates 